\title{
La conduite forcée de la chute de Pralognan
}

\section{The Pralognan Falls penstock}

\author{
PAR N. H. BEAUMEL

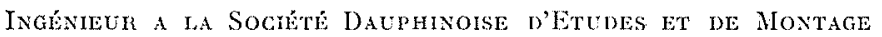

English synopsis p. 95

\section{Ly bassin de Pralognan et la nouvelle centrale de l'Electricité de France}

Pour se rendre à Pralognan, on emprunte la Route Nationale 90 de Grenoble à Aoste, sur le parcours d'Albertville à Moutiers, dans la vallée de la Tarentaise. A droite et à gauche de l'Isère se dressent des sommets de $2.500 \mathrm{~m}$. environ. L'un d'eux à droite, le Beaufortin, est un centre hydro-électrique important avec les usines de Venthon, Roenger, Queige, Villard, Beaufort, Fontanus, Hauteluce et Belleville (cette dernière alimentée par le lac de la Girotte), et les possibilités de cette région ne sont pas épuisées puis-

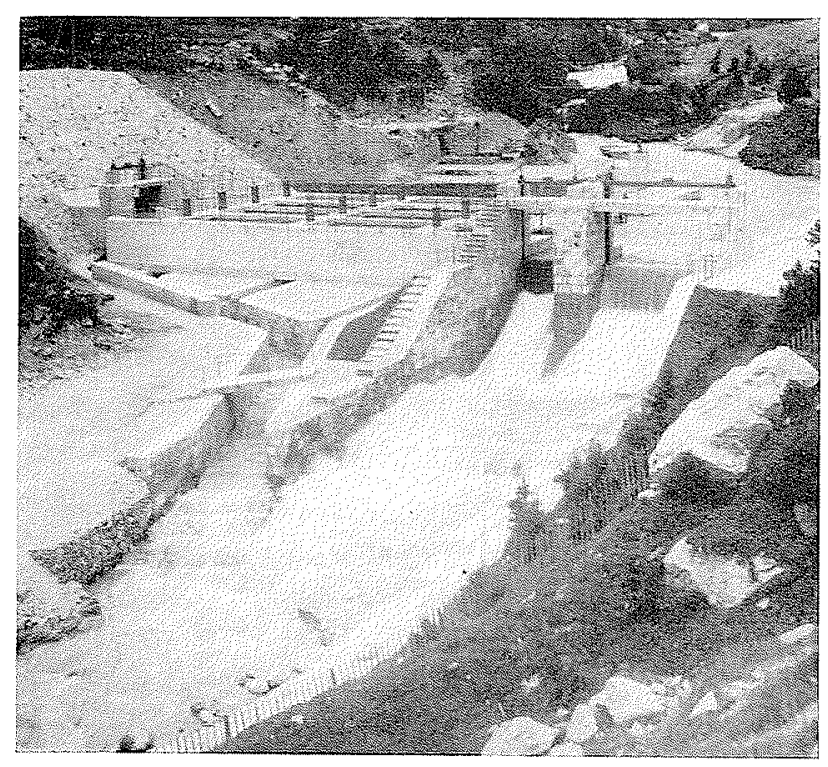

(Cliché Capellaro.)

PHOTO $\mathrm{N}^{\circ} 1$

Prise de Chavière vue pair l'aval. que 750 millions de kWh pourraient être fournis en restituant à l'Isère les eaux de Roselend.

Le bassin de Pralognan est moins bien équipé, les conditions nécessaires semblent cependant exister. A Moutiers, en effet, on se trouve à $480 \mathrm{~m}$. d'altitude et $28 \mathrm{~km}$ plus loin, à Pralognan, à $1.400 \mathrm{~m}$., devant le grand Massif de la Vanoise dont les sommets dépassent $3.600 \mathrm{~m}$.

Si l'on ajoute que la vallée est industrielle (il suffit de citer le nom de Bozel pour en faire foi), on entrevoit la possibilité d'une puissante utilisation hydro-électrique de la haute vallée. La centrale de Villard produisant annuellement 46 millions de kWh est loin d'exploiter ces possibilités, l'Electricité de France a donc mis en route, en 1949, la nouvelle centrale de Pralognan.

Cette centrale est équipée pour un débit de $8 \mathrm{~m} / \mathrm{sec}$. sous $724,80 \mathrm{~m}$ avec 3 alternateurs de $17.000 \mathrm{kVA}$ entraìnés par des turbines Pelton de $22.000 \mathrm{ch}$ à axe horizontal, tournant à $750 \mathrm{t} / \mathrm{mi}$ nute.

L'aménagement de cette chute avait été étudié par la Société Bozel-Malétra. Le premier projet, qui avait reçu un commencement d'exécution, a été repris par les services de l'Electricité de France et considérablement amplifié, si bien que l'usine, édifiée au confluent du Doron de Champagny et du Doron de Bozel, reçoit les eaux du Doron de Chavière, du ruisseau de l'Isertan, du torrent de la Glière et du ruisseau de la Vuzelle; sa productivité a été portée à 170 millions de kWh par an.

Le renforcement de la Conduite de Champagny

La nouvelle Centrale était implantée sur le tracé de l'ancienne conduite de Champagny, qui 


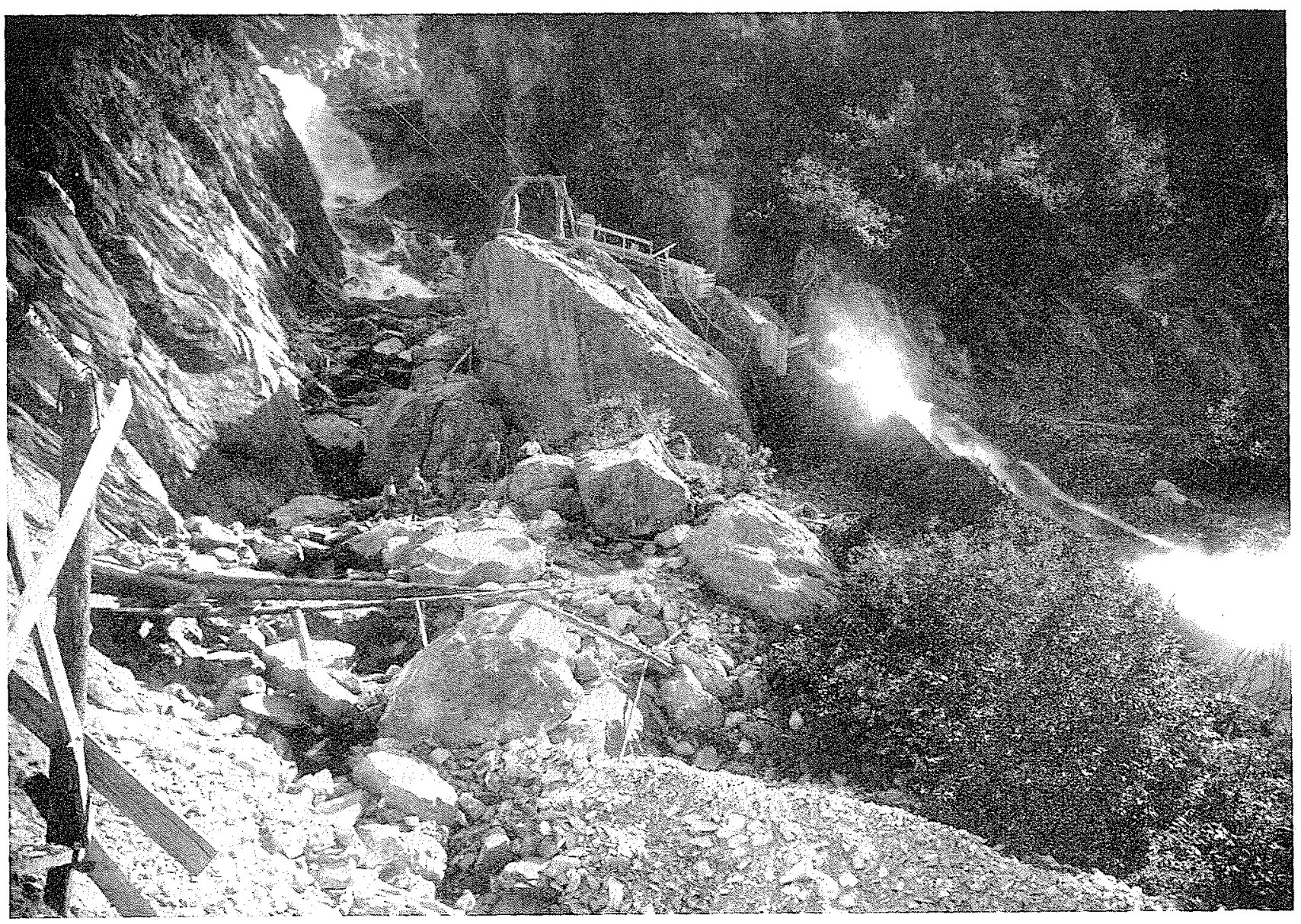

PHOTO Nं 2. -. SHE DE LA P'RISE DE R'ISERTAN.

(Cliché Capellaro.)

devait demeurer en service et alimenter la Centrale du Villard. Cette conduite avait été mise en place en 1911 et enterrée peu après. Les ingénieurs se sont demandés s'il était prudent, voire même acceptable, de laisser subsister dans les caves d'une Centrale électrique une conduite forcée d'un diamètre de $800 \mathrm{~mm}$ supportant une charge de $550 \mathrm{~m}$, après un service de 37 années.

Rappelons qu'à l'époque où fut installée la conduite forcée de Champagny on éprouvait les éléments en usine à une pression égale à une fois et demie la pression de service. Cette précaution s'est avérée insuffisante et depuis 1918 l'épreuve en atelier se fait au double de la pression maximum de marche. On s'est rendu compte, du reste, qu'un bon nombre de défauts étaient décelés à l'épreuve entre les valeurs 1,5 et 2 fois cette pression maximum de marche.

La conduite de Champagny ne donnait done pas toutes les garanties exigées par les normes actuelles; déjà au moment de son installation. La décision s'imposait donc : il fallait la renforcer. Le problème n'était pas facile à résoudre car il ne fallait pas interrompre l'exploitation de l'usine.

Après études, on procéda à des essais en atelier à la suite desquels le système suivant fut adopté : la conduite était renforcée à $30 \%$ par une série de frettes souples mises en tension par des amarrages à vis. Une élingue en acier à $200 \mathrm{~kg} / \mathrm{mm}^{2}$ de charge de rupture, analogue à celles employées sur les tuyaux à frettage souple (frettes individuelles), était conformée de facon à obtenir 2 brins parallèles raccordés par des boucles à chaque extrémité. Celles-ci, placées sur les deux éléments d'un serre-joint, pouvaient être rapprochées par le jeu d'une vis et d'un boulon visibles sur les photographies $n^{\circ} 4$ et 5 . on pouvait ainsi faire adhérer sur la tôle l'élingue et précontraindre la tôle jusqu'à ce que la fatigue du câble atteigne la valeur admise dans les calculs.

Le montage de ces dispositifs s'est effectué très facilement, sans aucune interruption de l'exploitation. Le monteur réglait la tension des frettes à l'aide d'un vérin hydraulique agissant sur les éléments rapprochables du serre-joint. Lorsque 
la pression sur les sabots atteignait la valeur désirée, il procédait au blocage des boulons.

Ce système de renforcement breveté est susceptible d'être employé dans d'autres cas.

\section{DONNÉES GÉNERALES}

Le plan de situation et le profil en long (voir fig. 1, p. 137) montrent la disposition des ouvrages.

La cote de retenue à la prise d'eau de Chavière est $1.642 \mathrm{~m}$; la cote de l'eau dans la chambre de mise en charge $1.619,80 \mathrm{~m}$ et la cote de restitution $895 \mathrm{~m}$. La hauteur de chute brute est donc de $725 \mathrm{~m}$ environ. La galerie d'amenée a une longueur de $12,5 \mathrm{~km}$ et une pente de $1,3 \mathrm{~mm}$ par mètre. La pression maximum de l'eau dans le collecteur atteint $830 \mathrm{~m}$.

Si l'on ajoute qu'il fallait prévoir l'évacuation depuis la chambre de mise en charge, d'un débit de $8 \mathrm{~m}^{3} / \mathrm{sec}$. (débit utilisé normalement par l'usine), nous aurons défini les bases qui ont présidé à l'étude de la conduite forcée.

\section{ETUde de IA CONDUITE FORCÉ DE L'USINe de Pralognan}

Bien que la puissance de $66.000 \mathrm{ch}$, installée sous $725 \mathrm{~m}$ de chute, demeure dans des limites éloignées du record de Bissorte $(105.000 \mathrm{ch}$ sous $1144 \mathrm{~m}$ de chute), la conception et l'installation de la conduite forcée et de la conduite de décharge de Pralognan n'allaient pas sans difficultés.

Les études commencées en 1943 avaient été menées dans le but de réduire au minimum le poids d'acier employé. A cette époque, en effet, plus qu'à toute autre encore, cette économie était précieuse. Aussi la solution en tuyaux soudés en acier à $48 \mathrm{~kg} / \mathrm{mm}^{2}$ de limite de rupture, qui conduisait à une dépense de $1.700 \mathrm{t}$. d'acier, fut-elle abandonnée au profit de la solution "légère » comportant des tuyaux soudés, puis surpressés, enfin auto-frettés. Le frettage prévu sur la plus grande longueur possible permettait de réduire le poids à 1.050 tonnes environ pour une longueur totale de $1.490 \mathrm{~m}$, soit un poids

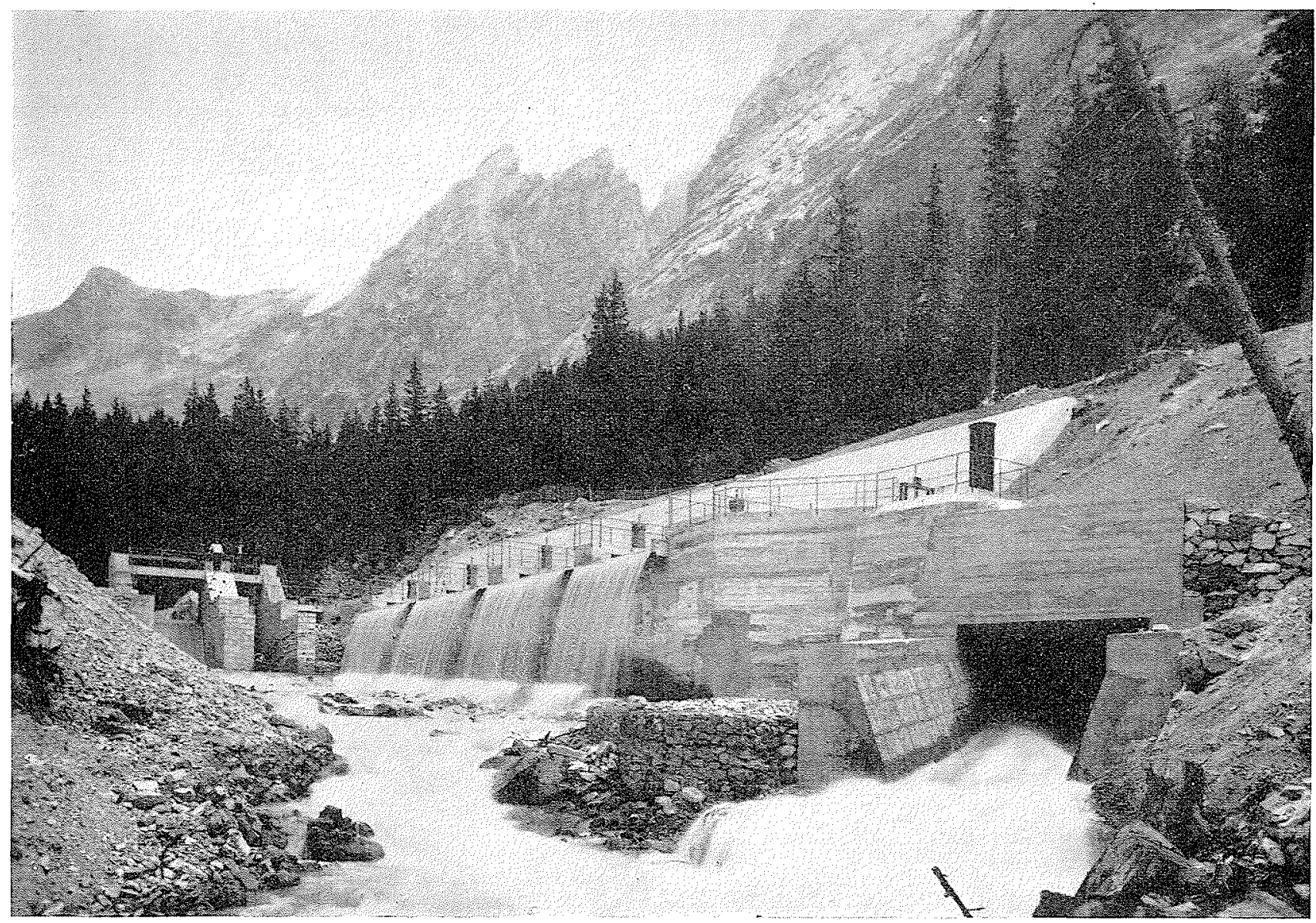

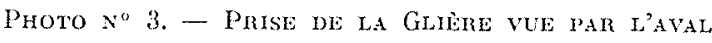


de $700 \mathrm{~kg}$ environ par mètre. En 1944, cette soIution était adoptée, les commandes de tôles prêtes à être enregistrées en forges.

La fabrication devait commencer en novembre 1946 et le montage se terminer en septembre 1947. Ces délais étaient imposés par les dates escomptées pour la réception des bons de monnaie-matière, puis de celle des matières premières.

En 1945, les relevés définitifs du terrain furent établis. De sérieuses difficultés d'implantation furent mises au jour et la longueur totale de la

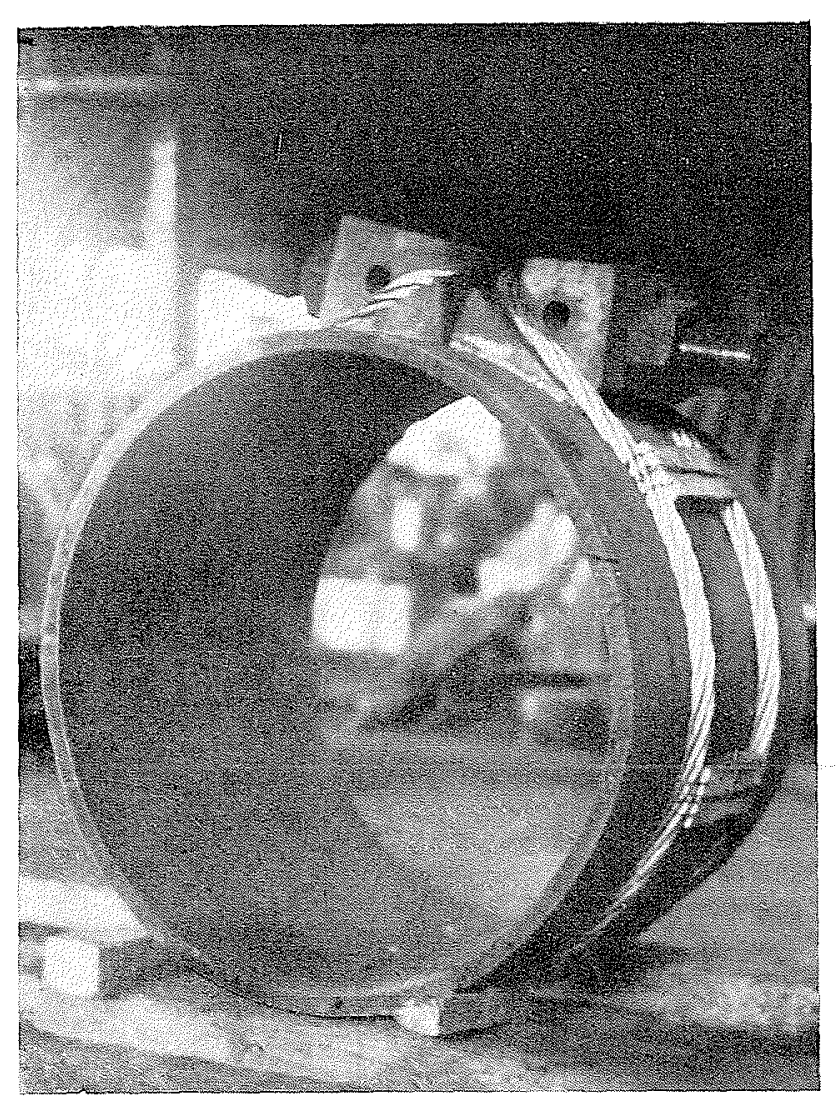

PHOTO No 4

conduite forcée dut ètre portée à $1.705 \mathrm{~m}$, collecteur non compris.

En décembre 1945, la question étant reconsidérée dans son ensemble, le poids de la fourniture était porté à 1.279 tonnes pour $1.705 \mathrm{~m}$. Cette solution comportait des tuyaux auto-frettés à frettage souple hélicoïdal. Ce système avait été mis au point entre temps. Sur la plus grande longueur, on avait prévu l'emploi de tôles d'importation américaine.

Mais la solution définitive ne devait voir le jour qu'en 1946 : l'emploi de tuyaux à frettage souple sur toute la longueur permettait d'abaisser

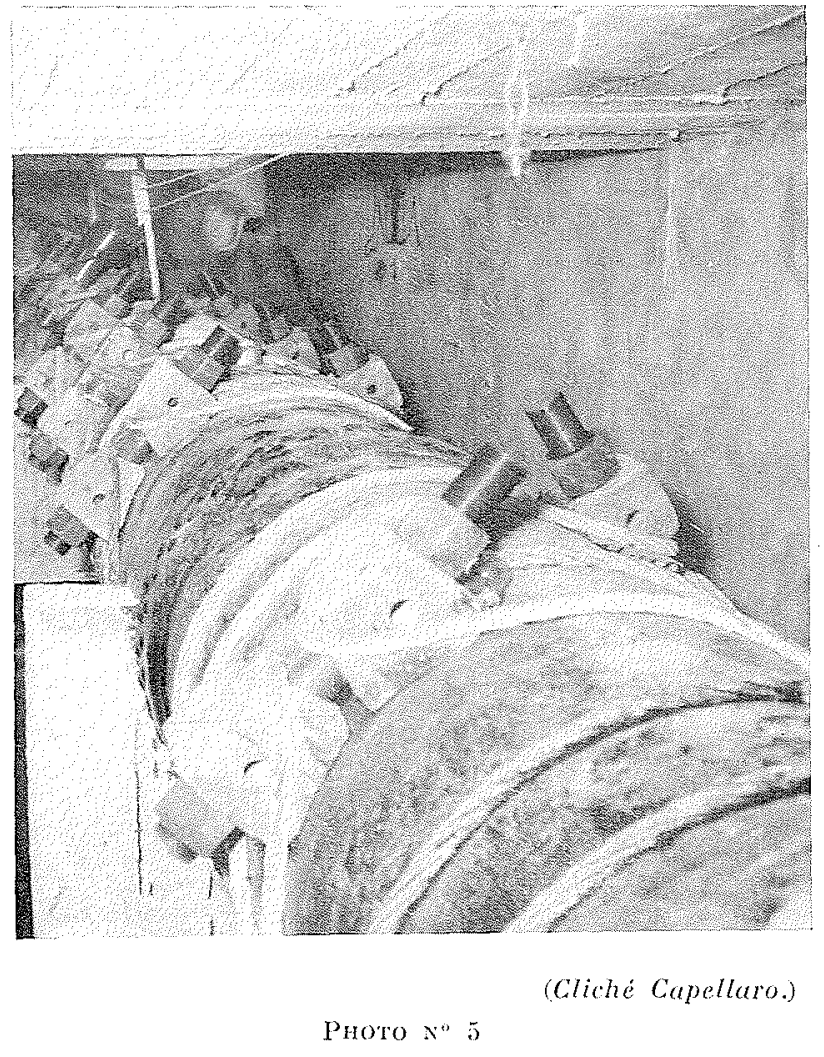

encore le poids total a 1.136 tonnes, soit un indice de poids au mètre de 665 , alors que l'indice normal pour une telle chute est voisin de 800 . Ce résultat était obtenu sans l'emploi d'acier au chrome-cuivre prévu dans les premières études, les forges ne pouvant plus assurer la fabrication de cet acier en 1946, faute de cuivre.

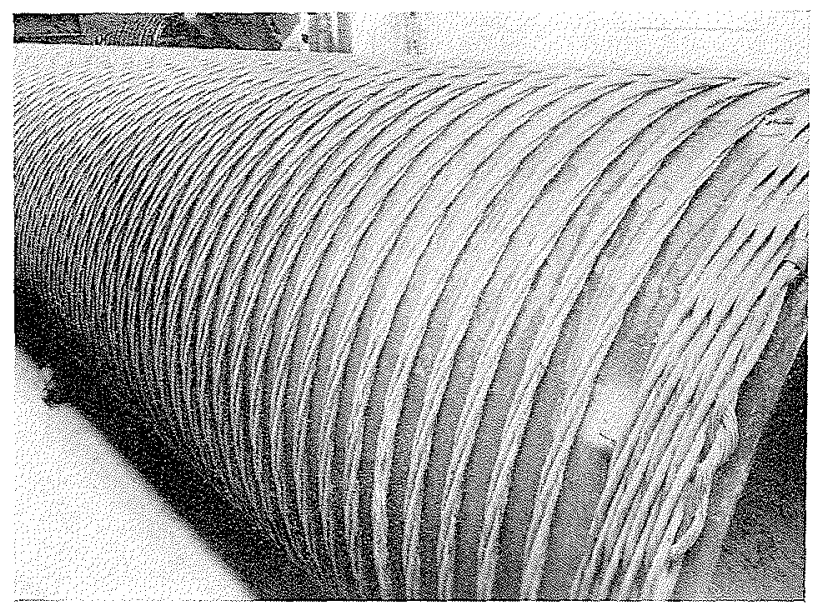

(Cliché Photopress.)

PHOTO No 6

TUYAU AUTO-FRETTE CABLE : détall. 


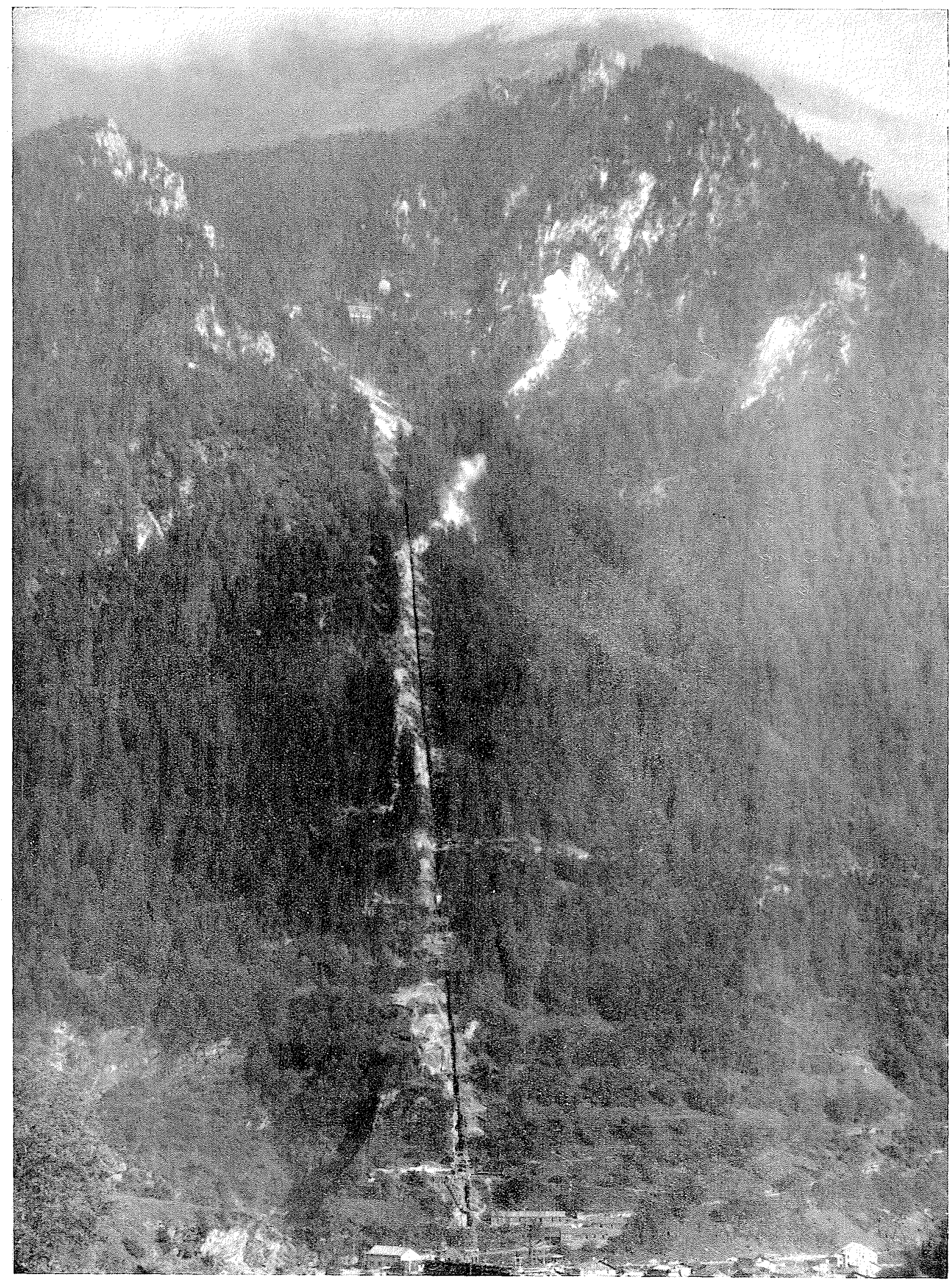

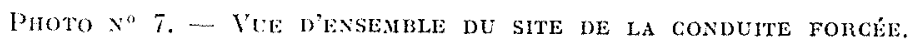

(Cliché Capellaro.) 
En fait, on utilisa sur une certaine longueur les tôles de provenance américaine qui depuis 1945 faisaient couler beaucoup d'encre et modifier un grand nombre de spécifications techniques, et quand les dessins d'exécution furent approuvés, le poids total atteignait 1.180 tonnes pour une longueur de $1.705 \mathrm{~m}$.

Le tableau ci-après précise le mode de construction des différents tronçons.

Condute forcée. - Caractéristiques.

\begin{tabular}{|c|c|c|c|c|c|c|c|c|}
\hline & Cote & 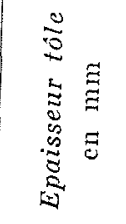 & 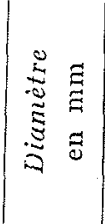 & 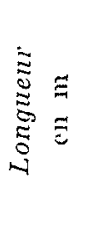 & $\begin{array}{c}\text { Pente mètre } \\
\text { pal mèlre }\end{array}$ & $\begin{array}{l}\text { Frettage } \\
\text { en mm }\end{array}$ & 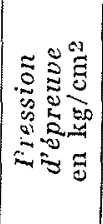 & 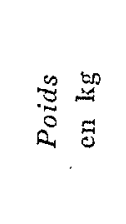 \\
\hline $\begin{array}{c}\text { T.S. } 50 \\
\text { Tuyaux soudés }\end{array}$ & 1.619 à $1.54 \bar{\jmath}$ & 6 et 7 & 1.450 & 141 & 0,449 à 0,881 & & & 39.465 \\
\hline $\begin{array}{c}\text { T.A.F. } 50 / 200 \\
\text { Tuyaux à frettage } \\
\text { souple hélicoïdal.. }\end{array}$ & 1.545 à 1.243 & 6 à 11 & 1.450 & 786 & 0,106 à 0,707 & $\left\{\begin{array}{c}\text { câble: } 4 \times 7 \times 2,4 \\
\text { pas: } 55 \text { à } 70\end{array}\right.$ & $\begin{array}{c}50 \\
\dot{d} \\
98\end{array}$ & 343.965 \\
\hline $\begin{array}{l}\text { T.A.F. } 50 / 90 \\
\text { Tuyaux à frettage } \\
\text { rigide . . . . . }\end{array}$ & 1.243 à 897 & 9 a 25 & 1.300 & 773 & 0,0 à 0,890 & $\left\{\begin{array}{r}\text { frettes : } 60 \times 17 \\
\dot{a}: 70 \times 31 \\
\text { pas: } 60 \text { et } 70\end{array}\right.$ & $\begin{array}{c}98 \\
\dot{a} \\
200\end{array}$ & 676.483 \\
\hline Accessoires . . . . . . & & & & & & & & 30.603 \\
\hline Collecteur & & & & 32 & & & & 98.733 \\
\hline \multicolumn{3}{|c|}{ Longueur totale } & \multicolumn{2}{|c|}{$1.732 \mathrm{~m}$} & \multicolumn{2}{|c|}{ Poids total } & \multicolumn{2}{|c|}{$1.189 .249 \mathrm{~kg}$} \\
\hline
\end{tabular}

\section{Caractéristroues de ra condutte Forcée}

$1^{\circ}$ Les tuyaux. - Il n'est pas utile de décrire les tuyaux T.A.F. $50 / 90$ à frettes rigides, employés industriellement depuis près de vingt ans. Quant aux premiers tuyaux à frettage souple, ils furent fabriqués en 1943 pour équiper la chute de Vénéon $\left(H=400 \mathrm{~m}, Q=15 \mathrm{~m}^{3} / \mathrm{sec}\right.$.) étudiée en 1940 . A cette époque, l'approvisionnement des frettes laminées était difficile et il fut envisagé de les remplacer par des câbles à torons. Ceuxci présentaient un avantage considérable en ce qui concerne l'économie de poids, puisque la limite de rupture du câble pouvait être égale à $200 \mathrm{~kg} / \mathrm{mm}^{2}$ alors que celle de l'acier laminé et traité n'était égale qu'à $90 \mathrm{~kg} / \mathrm{mm}^{2}$. Toutes choses égales d'ailleurs, une frette d'acier tréfilé de $450 \mathrm{~mm}^{2}$ de section avait la même résistance de rupture qu'une frette d'acier laminé de $1.000 \mathrm{~mm}^{2}$ de section.
La fabrication d'une telle frelte n'était pas aisée, car l'acier en fils ne peut ni se forger, ni se souder sans perdre ses qualités. Elle fut entreprise cependant avec succès, en partant de câbles toronés à 7,12 , .. etc. fils, de diamètres au plus égaux à $3 \mathrm{~mm}$.

Le toronage est exécuté en 6 passes autour d'un gabarit. En fin d'opération, le câble dont on réserve des longueurs convenables est introduit à la place du gabarit de façon que toutes les sections soient composées de 7 torons dont 1 central. Au point de jonction, la section ne comporte que 6 torons, aussi la résistance de la frette souple retenue dans les calculs n'est-elle que les $6 / 7$ de la section totale d'acier. Cette imperfection devait conduire à l'invention du câblage hélicoïdal qui consiste à poser sur un tuyau, sans tension, un câble enroulé avec un pas déterminé. Le frettage s'opère, après ligature des extrémités, comme pour les tuyaux à frettes individuelles. Sous l'ef- 

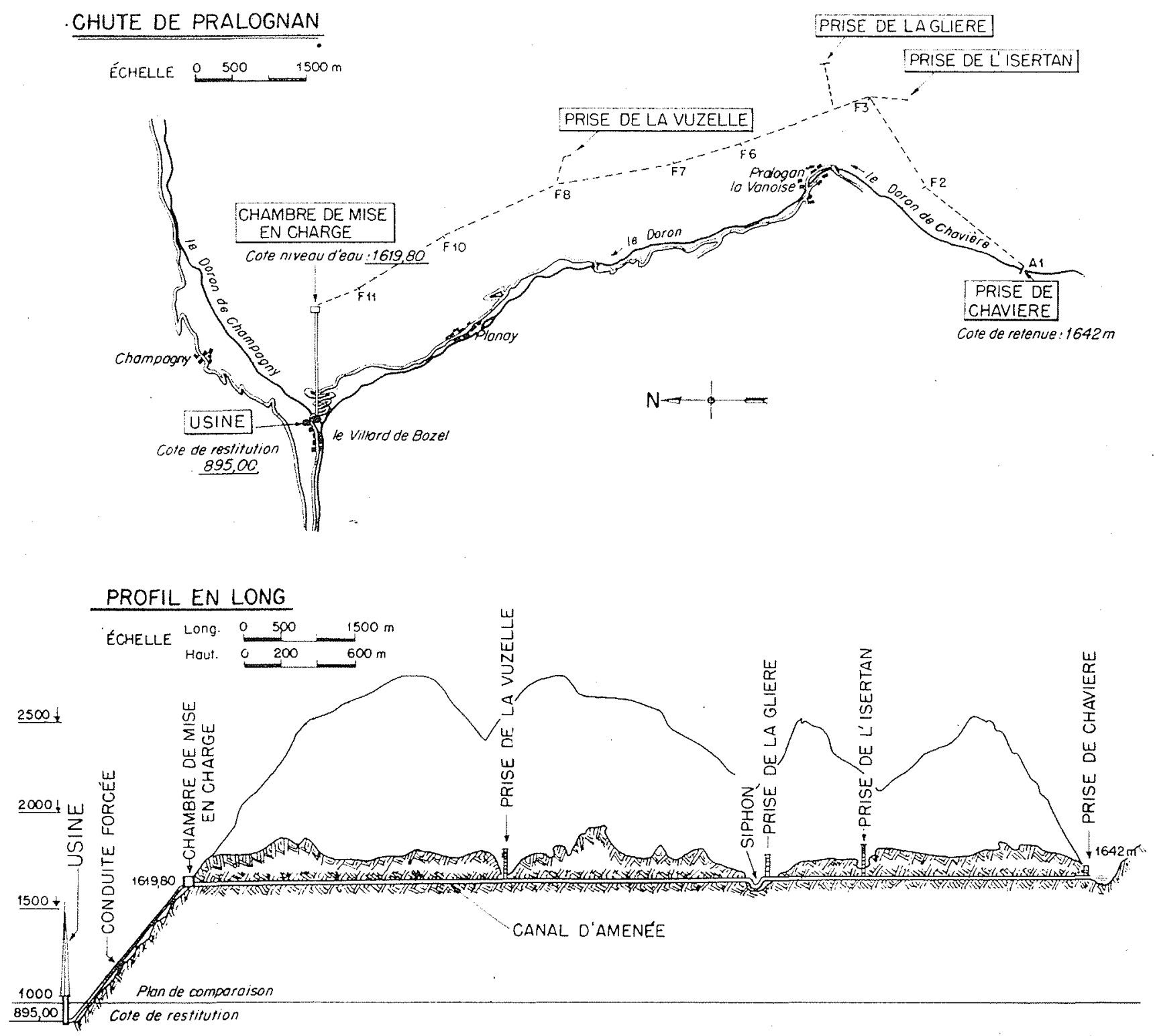

Fig. 1. - Plan de situation. Profil en long.

fet de la presse hydraulique, une pression intérieure croissante s'établit, la paroi d'acier dépasse sa limite élastique et acquiert une déformation permanente, en sorte que sous une pression nulle, la tôle soit précontrainte et qu'elle travaille à un taux au plus égal à sa limite élastique quand on atteint 2,5 fois la pression maximum de service.

De plus, dans les tuyaux à frettage hélicoïdal, on détermine les sections résistantes de tôle et de câbles de telle façon que si, pour une cause quelconque, le frettage était détruit, la paroi puisse résister à la pression maximum de service, avec un coefficient de sécurité au moins égal à 1 .
Le tuyau ainsi concu satisfait à la fois le technicien avide de rendement et l'utilisateur à la recherche de sécurité.

2" Le tracé de la conduite et le montage. Le tracé de la conduite forcée a été assez difficile à déterminer. Il fallait, en effet, traverser des zones d'éboulis, dans la partie haute, et la route nationale 515 qui monte en lacets au-dessus de Villard-de-Bozel où est implantée l'usine. Un grand nombre de sujétions intervenaient et on ne peut affirmer que la solution choisie fut la meilleure car certaines difficultés n'apparurent qu'au moment des fouilles ou du montage.

Dans tous les passages particuliers et dans la 
forêt au-dessus de Villard (classée par la Commission des sites), la conduite est enterrée. Plus haut, les tuyaux à fretlage hélicoïdal sont enterrés sur $226 \mathrm{~m}$ et à l'air libre sur $312 \mathrm{~m}$ et $248 \mathrm{~m}$.

La partie enterrée se trouve sur un couloir d'éboulis, condition encore aggravée ver's le haut par le fait d'une forte pente en travers. La tranchée fut difficile à exécuter et la conduite dut être protégée après monlage par des murs de pierre sèche.

Tous les $200 \mathrm{~m}$ environ des trous d'homme ont été établis pour permettre la visite du revètement d'émail bitumineux à lintérieur de la conduite. Ce revêtement a été appliqué à chaud à pied d'cuvre. Un atelier de peinture avait cté aménagé pour procéder à cette application avant le montage des éléments. Actuellement on procède de préférence à l'émaillage en usine, qui garantit des résultats plus homogènes.

La couche d'émail bitumineux doit permette, en même temps que la protection contre la corrosion, la stabilisation des pertes de charges. Cel-
Ies-ci ont été évaluées, pour un débit de 8.000 litres par seconde, à $48,166 \mathrm{~m}$, soit à $6,6 \%$ de la chute.

Les pertes de charges linéaires ont été calcubées en appliquant la formule de Maurice Léry :

$$
J=\frac{V^{2}}{420,25 \cdot R(1+3 \sqrt{R})} \times 0,75
$$

0,75 est un coefficient adopté pour les tuyaux soudés.

Avec des vitesses du fluide égales respectivement à $4,846 \mathrm{~m} / \mathrm{sec}$. et $6,029 \mathrm{~m} / \mathrm{sec}$, on arrive à un total des pertes linéaires de $37,050 \mathrm{~m}$.

On reproche à la formule de $M$. Lévy d'être ancienne el empirique, mais les formules modernes laissent aussi place à des approximations, pour estimer la rugosité par exemple, si bien qu'on hésite a les adopter, d qu'on préfire conserver la formule de Lévy dont les résultats sont confirmés par lexpérience depuis de nombreuses

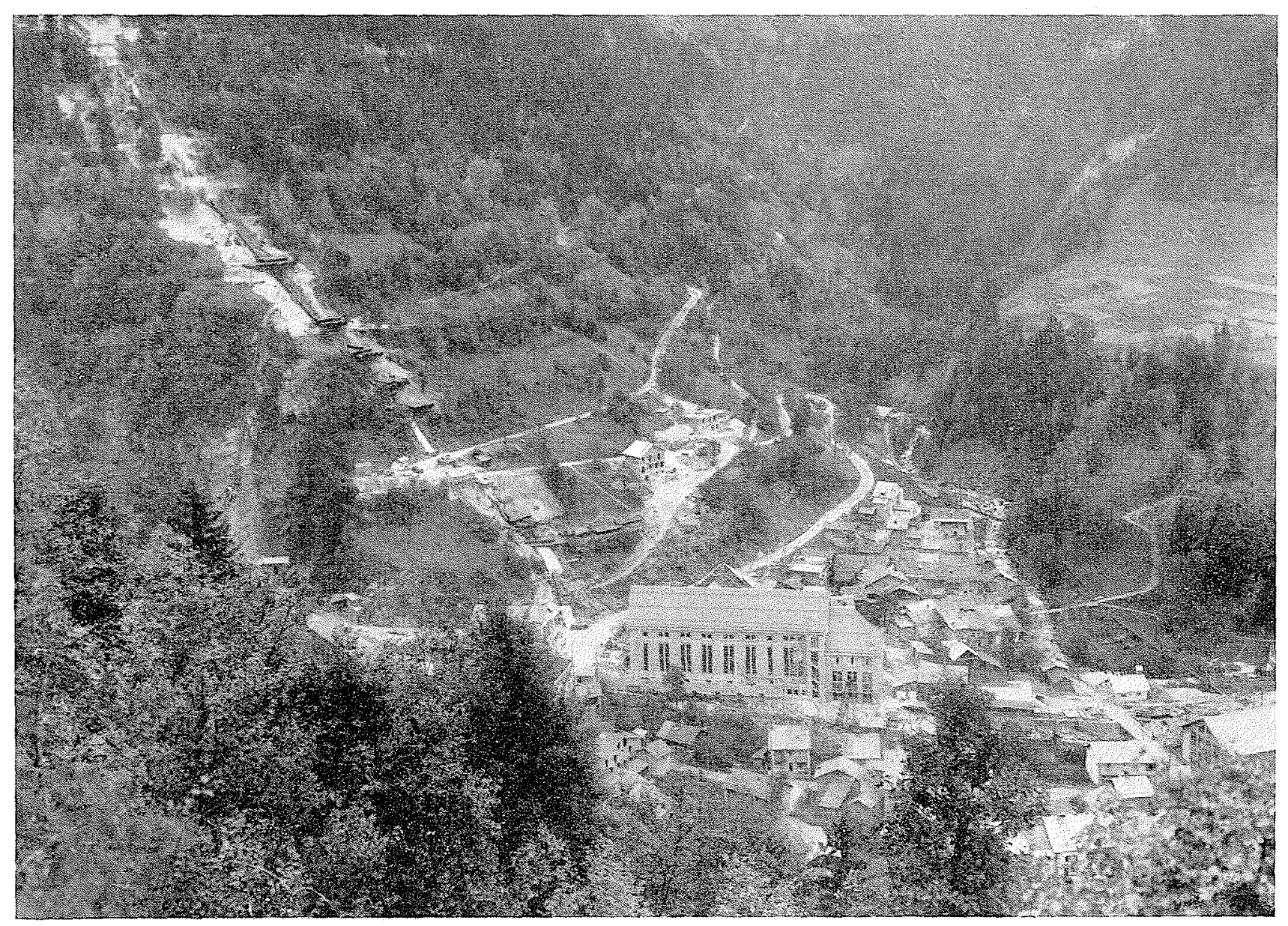

Photo $x^{\circ}$ 8, - La vovverle cextrale.

On distingue la conduite forcée et la conduite de décharge dans leur partie basse ainsi que les stocks de tuyaux en attente. 


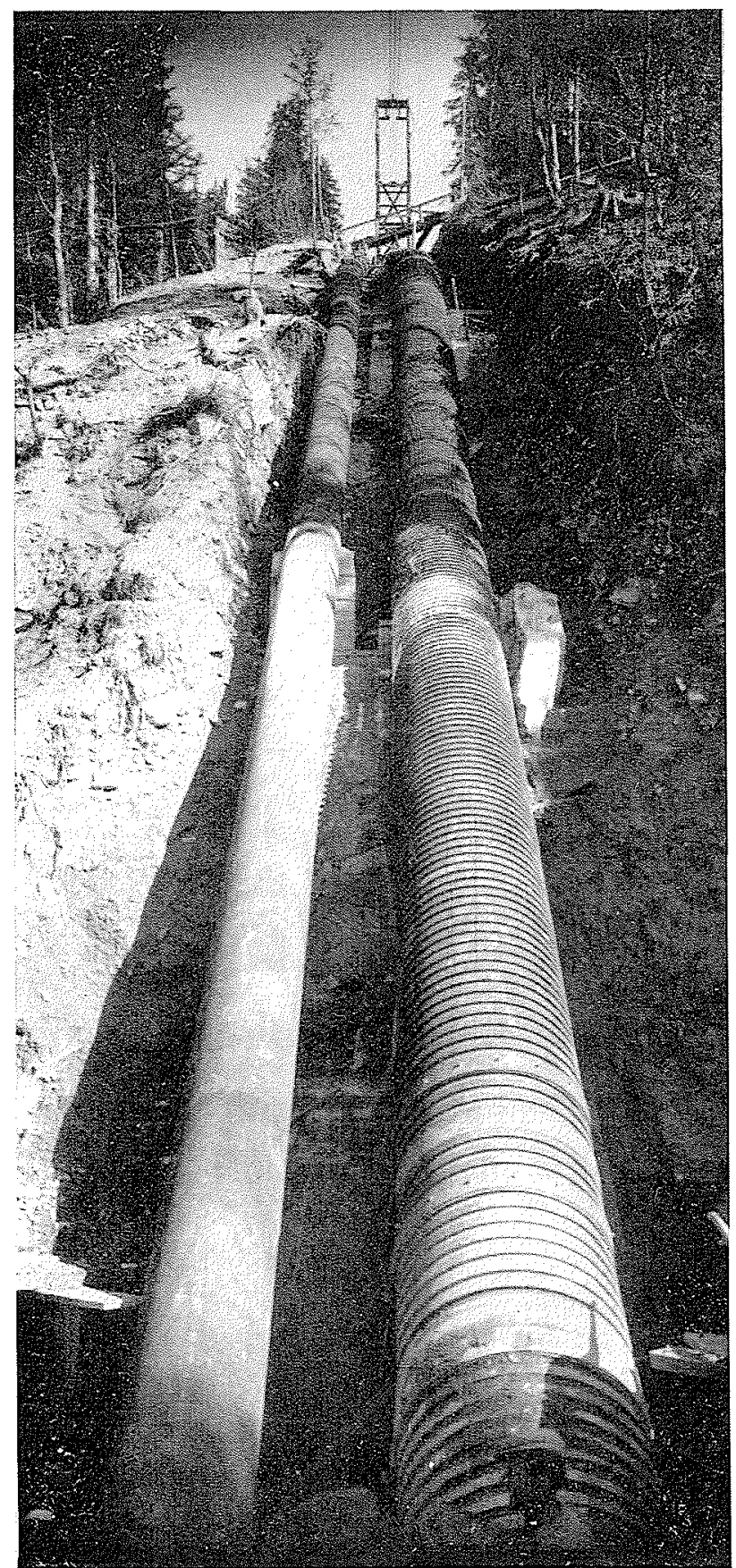

(Gliché Capellaro.)

PHOTO N० 9

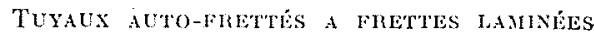

années. Elle a le mérite de donner de bons résultats, par exces, pour les vitesses de l'ordre de 3 à 5 mètres par seconde dans des diamètres de l'ordre de $1.500 \mathrm{~mm}$.

Les pertes de charges dans le collecteur sont égales à $4,593 \mathrm{~m}$ dont $3,334 \mathrm{~m}$ dans les 6 tés de bifurcation en acier coulé. Ces pièces sont assembles aux tuyaux ato-frelles constituant les parties rectilignes, par des joints a brides boulonnées. L'ensemble du collecteur est bloqué dans la maconnerie à l'exception des pieces de bifurcation.

La conduite forcée, en plus du collecteur, esl bloquée par des massifs de maconnerie disposés à tous les coudes. Elle repose entre les massifs, sur des pilier's supports, disposés tous les 9,50 m environ.

Les fonilles nécessaires pour ces massifs ont conduit à enlever un volume de $4.000 \mathrm{~m}^{3}$ de déblais. Les terrassements pour l'exécution de la tranchée ont été beaucoup plus importants et ont dépassé $21.000 \mathrm{~m}^{3}$.

D'autre part, $4.800 \mathrm{~m}^{3}$ de béton ont été mis en ouvre pour assurer la stabilité de la conduite forcée. Ce chiffre est relativement considérable pour deux raisons : le terrain rencontré n'était pas de qualité excellente et la pression sur le sol devait rester inférieure à $2 \mathrm{~kg} / \mathrm{cm}^{2}$ chaque fois que les fouilles ne mettaient pas à jour le rocher; de plus, la présence d'une route nationale s'élevant en lacets et coupée par la conduite obligeait à augmenter le nombre des condes et celui des massifs.

$3^{\circ}$ La décharge. - - Dans le chiffre ci-dessus est aussi compris le béton utilisé pour les ouvrages assurant la stabilité de la conduite de décharge. Celle-ci doit permettre le débit de la galerie à écoulement libre, soit $8 \mathrm{~m} \% / \mathrm{sec}$. Elle est placée parallèlement à la conduite forcée $(\varnothing 1.450,1.100,950$ et $800 \mathrm{~mm}$ ), sauf dans la partie basse où elle rejoint par le plus court chemin le Doron de Champagny. Pour sa fabrication, on a employé des tôles d'acier SiemensMartin au carbone, extra-doux, ayant une résistance de rupture minimum de $33 \mathrm{~kg} / \mathrm{mm}^{2}$, une limite élastique de $22 \mathrm{~kg} / \mathrm{mm}^{2}$, alors que pour l'acier demi-doux supérieur, employé pour la conduite forcée, la limite élastique est supérieure à $28 \mathrm{~kg} / \mathrm{mm}^{2}$. Les dimensions de la conduite de décharge ont été déterminées de façon à ce que l'écoulement de l'eau puisse toujours se faire avec surface libre, d'une part, et que les tuyaux puissent résister au vide intérieur. En usine, ces tuyaux ont été éprouvés à une pression telle que la fatigue de la tôle atteigne $16 \mathrm{~kg} / \mathrm{cm}^{2}$. Pour la conduite forcée, au contraire, comme nous l'avons indiqué au début, tous les tuyaux sont construits de façon que, sous 2,5 fois la pression maximum de service, la fatigue de la tôle soit inférieure ou au plus égale à sa limite élastique. Ils sont éprouvés en usine sous une pression au moins égale à deux fois la pression maximum de service (charge statique $+15 \%$ ). 


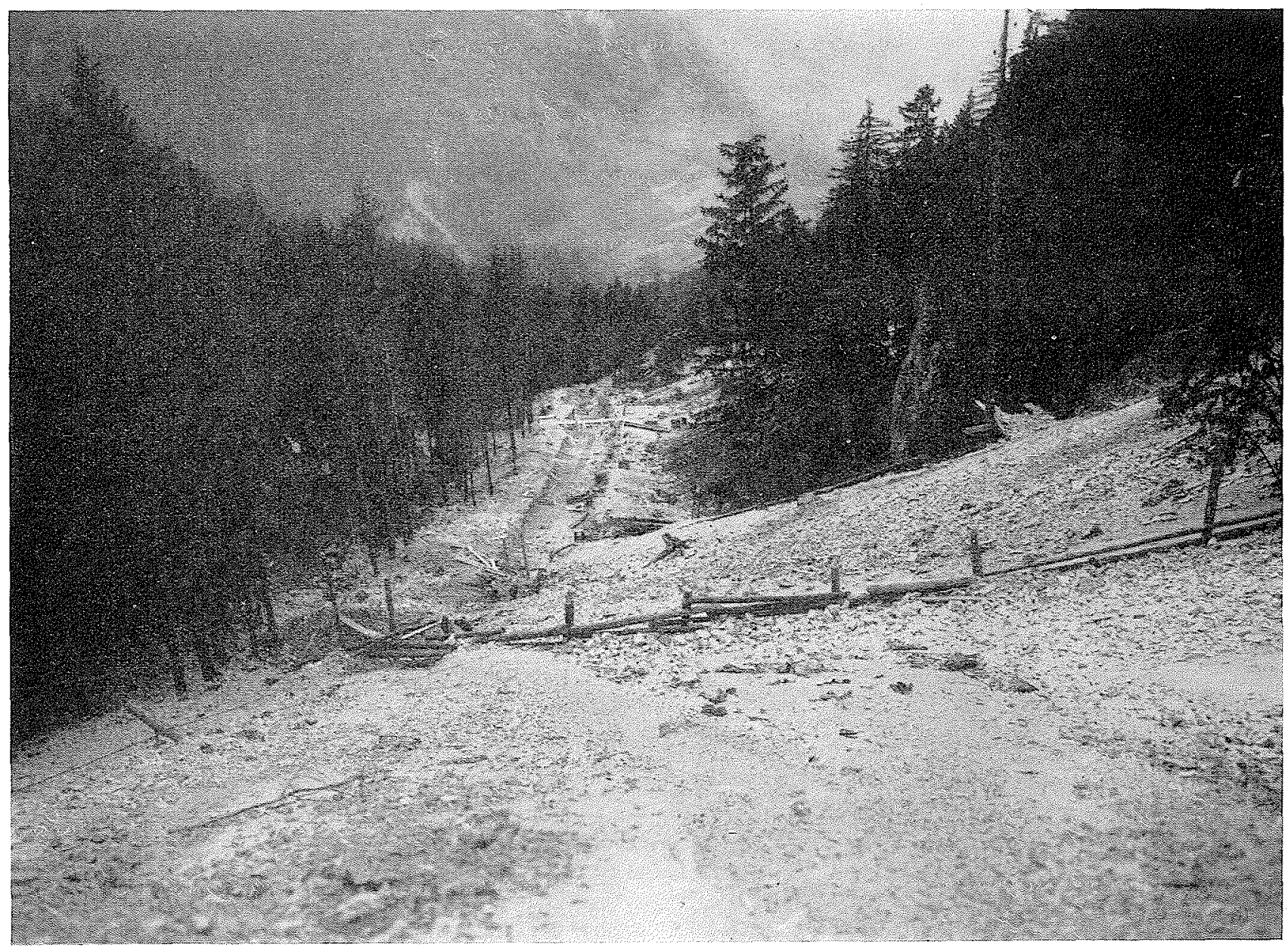

PHOTO $N^{2}$ 10. - - LA ZONE D'BBOULIS AU MOMENT DES FOUILIES.

(Cliché Baranger.)

4" Les dispositifs de sécurité et les essais. La conduite forcée doit aussi résister au vide intérieur qui est toujours à craindre, bien qu'à la sortie de la chambre de mise en charge, à l'aval du robinet, soit dispose un reniflard permettant l'entrée de l'air en cas d'arrêt brusque du débit de la conduite. La fermeture du robinet est normalement commandée depuis l'usine avec précaution; cependant, en cas d'accident, le débit est automatiquement et brusquement arrêté par l'action d'une palette, laquelle, sous l'effet d'une grande vitesse de l'eau, s'incline jusqu'à déclancher un dispositif de sécurité.

Après achèvement du montage, la conduite a été éprouvée avec une surpression de $15 \%$ au collecteur où la pression élait donc de $83,1 \mathrm{~kg} / \mathrm{cm}^{2}$; à proximité du fond plein, soudé provisoirement à l'amont, elle était de $12,5 \mathrm{~kg} / \mathrm{cm}^{2}$, ce qui rem présente une surpression considérable pour les tuyaux de la partie supérieure. L'essai à surpression constante éprouve la stabilité de la conduite dans le cas le plus défavorable de surpres- sion accidentelle. Ces essais n'ayant donné lieu à aucune observation, les soudures ont été alors martelées.

\section{LE TRANSPORT DES MATÉRIAUX}

Le transport des éléments s'est effectué depuis l'atelicr du constructeur à Grenoble, par camion jusqu'à pied d'ouvre, et de là, à l'aide principalement d'un téléférique, jusqu'au lieu d'emploi.

Ce téléférique devait assurer la manutention de tuyaux pesant jusqu'à 10 tonnes et d'un encombrement maximum de $9,600 \mathrm{~m}$ de longueur, et $1,700 \mathrm{~m}$ de diamètre. Il devait, en outre, assurer le transport des matériaux nécessaires aux travaux du Génie civil, par charges de 7 tonnes au maximum.

La pente moyenne de cette installation était de $44 \%$ pour une longueur, entre ancrages, de $1.534 \mathrm{~m}$, reposant sur 5 pylones; la plus longue portée atteignait $432 \mathrm{~m}$. 
Cette installation fut montée entre le $1^{\text {er }}$ septembre 1946 et le 20 juillet 1947. Les deux câbles porteurs, de $45 \mathrm{~mm}$ de diamètre, furent déroulés à la main, au cours d'un mauvais hiver et au milieu de toutes sortes de difficultés.

Le câble tracteur sans fin, de $24 \mathrm{~mm}$ de diamètre, était actionné par un treuil situé à l'ancrage inférieur. Le câble moufleur, de $18 \mathrm{~mm}$ de diamètre, permettant les mouvements verticaux de la charge, était commandé par un treuil ancré à la partie supérieure.

Tous ces câbles élaient composés d'une âme textile et de 6 torons d'acier à $180 \mathrm{lig} / \mathrm{mm}^{2}$ de limite de rupture.
La réunion des deux extrémités du câble tracteur s'opérait sur l'attelage du chariot porteur, constitué par 2 boggies à 8 roues à axes horizontaux articulés sur une poutre d'accouplement en treillis.

Cette installation très robuste a permis le montage de 90 tonnes de tuyaux par mois en moyenne, depuis novembre 1947 jusquau printemps 1949. Dans la partie haute, le montage s'est effectué à l'aide du plan incliné, en partant de la chambre des vannes (hyau $n^{\circ} 225$ ) jusqu'au tuyau $n^{\circ} 219$, puis en partant du $n^{\circ} 136$ jusqu'au tuyau n" 218.

Bien que le montage n'ait pas été exécuté à

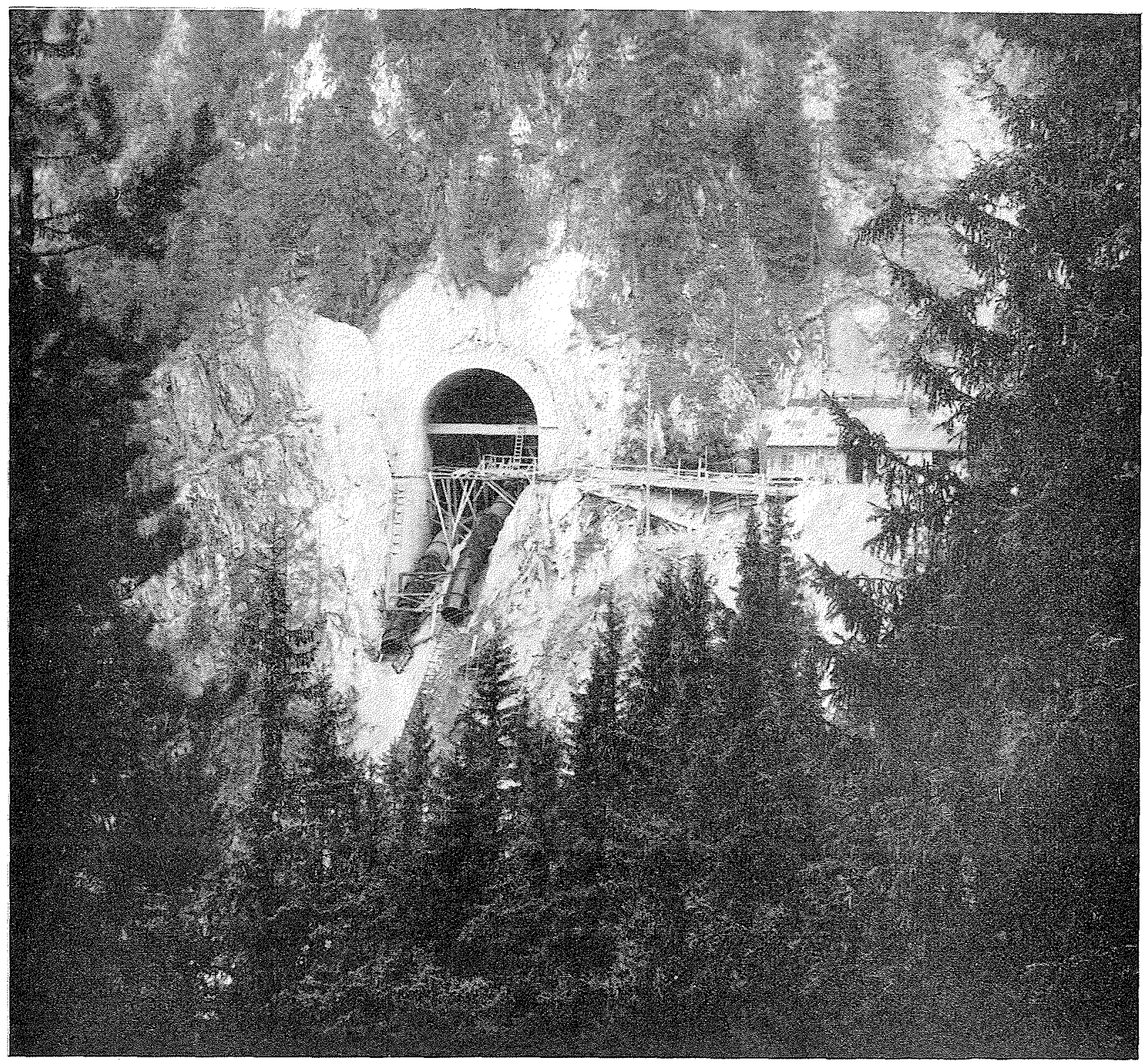




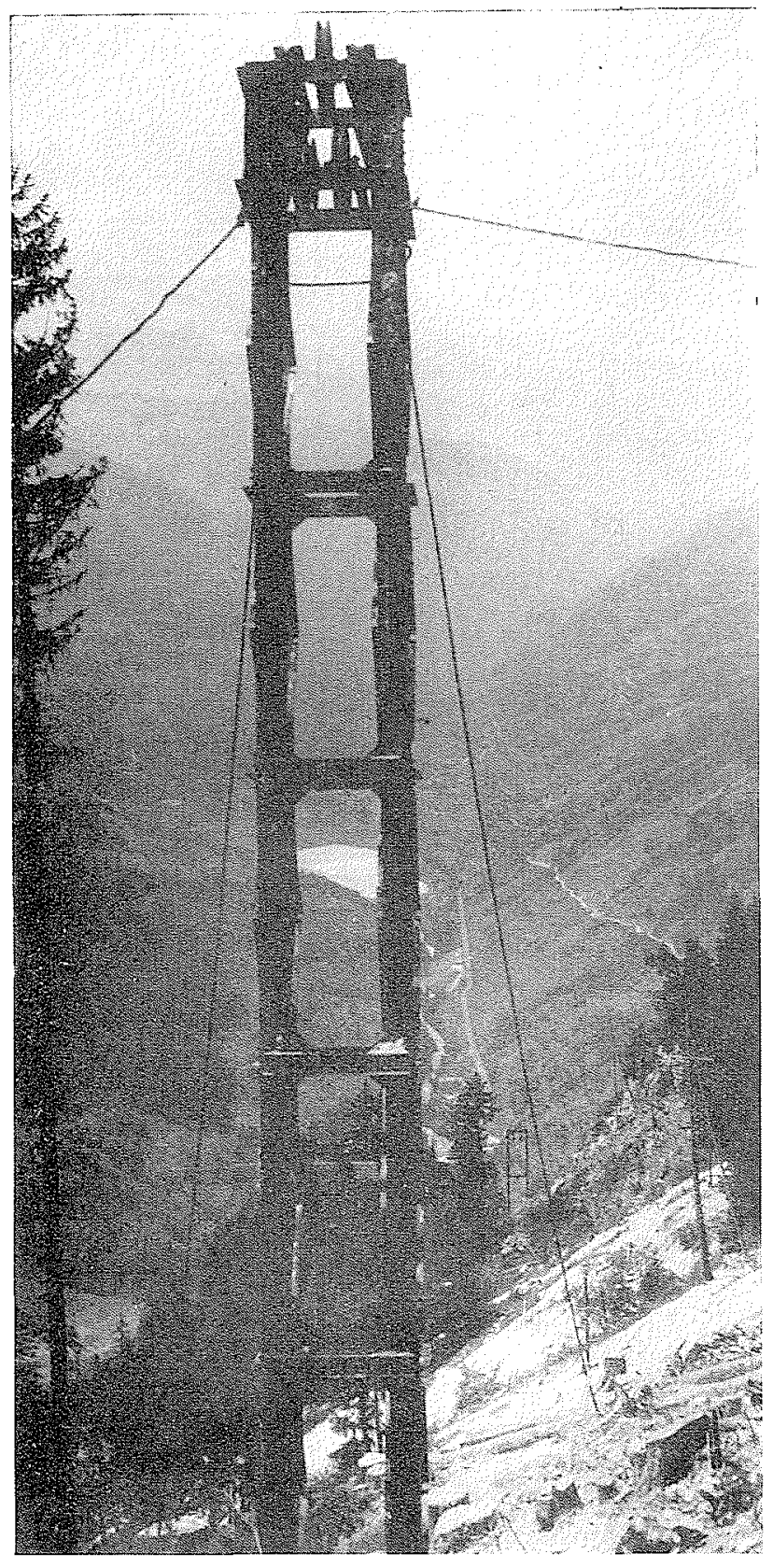

PHOTO N० 12

(Cliché SDEM.)

IE PYLONA DE RENYOA DV TELEPHERTQUE.

une cadence accélérée, des problèmes de stockage se sont posés. La présence d'un téléférique a grandement facilité ces opérations : les tuyaux étaient entreposés sur des estacades, puis mis en peinture et stockés une dernière fois, le long du profil.

\section{L'ACHEVEMENT ET L'INAUGURATYON}

Depuis l'achèvement des différents troneons jusquà la mise en eau, la conduite forcéc a pu se dilater librement sous l'effet des variations de température, tant que les manchettes de raccordement sont restées libres. Tous les $200 \mathrm{~m}$ environ est placée une virole dont le diamètre intérieur est égal au diametre extérieur du chemisage des tuyaux. Elle sert de raccordement coulissant et peut ètre ajustée à la longueur exacte séparant le point théorique de départ d'un troncon amont avec le point pratique d'arrivée du tronçon immédiatement à l'aval. Cette manchette est soudée normalement sur le tuyau amont et laissée libre sur le tuyau aval. Lorsque le montage est achevé, les dernières soudures de raccordement sont effectuées à une température telle qu'en aucun cas, sous l'effet d'un grand froid ou d'une forte chaleur, les tensions longitudinales créées par la dilatation ou la contraction de l'acier ne dépassent le maximum admissible.

Dans la partie basse du profil, ces manchettes coulissantes sont remplacées par des joints à brides, distants de quelques centimetres. Un fauxjoint est usiné à une côte telle qu'après sa mise en place les conditions d'exploitation soient satisfaites.

La mesure de la température d'une conduite forcée est une opération assez délicate, aussi

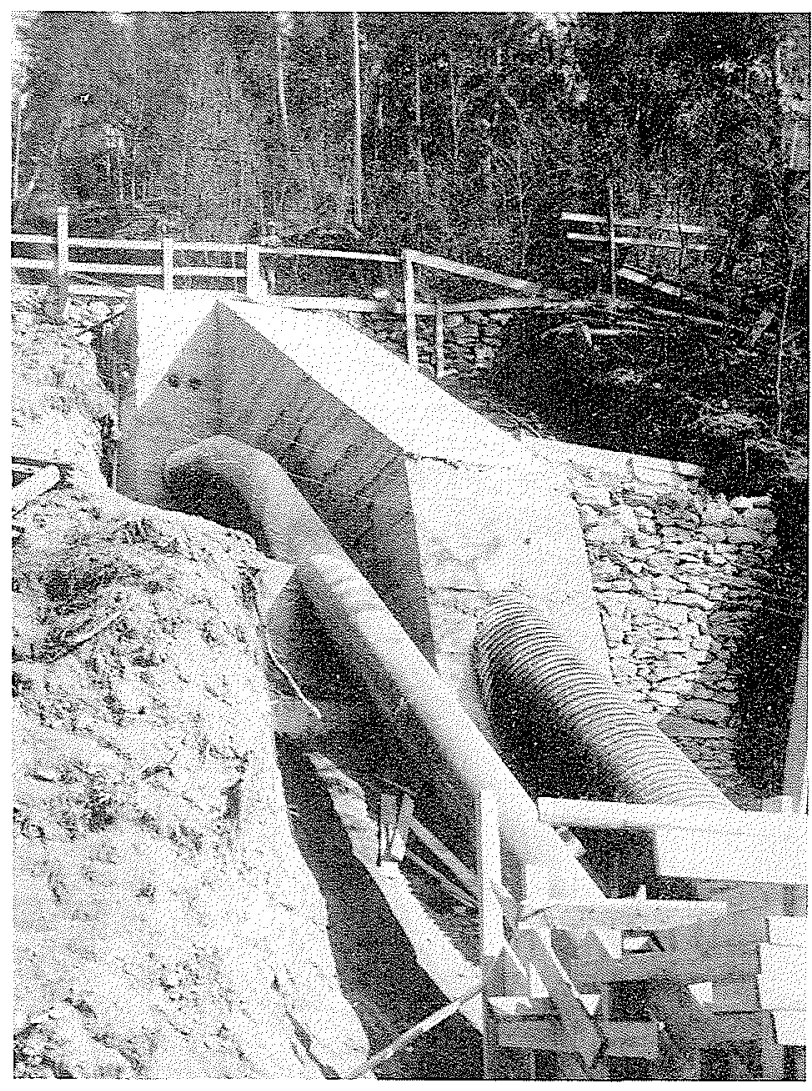

(Cliché Capallaro.)

Pното No $1:$

UN passage sous la roUte nationale. 
n'est-elle pas laissée à l'initiative pure et simple des monteurs. L'intervention des services techniques permet d'opérer les raccordements dans les meilleures conditions. En 1949, à Pralognan, il a fallu attendre assez longtemps le bon vouloir de la nature pour pouvoir bloquer la conduite forcée à la température prédéterminée. Lorsque les circonstances ne permettent pas cetle atiente, on peut utiliser un procédé breveté qui permet d'immobiliser la conduite à n'importe quel moment en Iui appliquant, dans le sens longitudinal, des tensions appropriées.

L'inauguration de la Centrale eut lieu le $16 \mathrm{sep}-$ tembre 1949. La conduite forcée avait été mise en eau le 8 juin, éprouvée le 14 juin.

Le 18 novembre, toutes les installations étaient démontées et les ouvriers quittaient Villard-deBozel où ils avaient travaillé pendant trois années.

Aujourd'hui oì la France a retrouvé les possibilités industrielles d'antan, il semble que la même tâche pourrait être menée à bien dans un plus court délai. Il n’en est pas moins varai que l'aménagement de la chute de Pralognan a nécessilé, de la part des exécutants comme du Maitre de l'CEuvre, un labeur patient et tenace auquel nous nous devons de rendre hommage.

Ces quelques lignes n'ont d'ailleurs pu faire pressentir qu'une partie du travail accompli pour l'édification de cet ouvrage, lequel n'est lui-même qu'un simple épisode de l'histoire de l'Energie Electrique en France. 